\title{
Untersuchungen zur Synchronisation von Herz- frequenz und musikalischem Rhythmus im Rah- men einer Entspannungstherapie bei Patienten mit tumorbedingten Schmerzen
}

\author{
U. Reinhardt \\ Ambulanz für Hämatologie und Onkologie, Klinikum Bayreuth
}

\section{Schlüsselwörter}

Musiktherapie · Entspannungstherapie · Rhythmusinteraktionen · Schlafstörungen $\cdot$ Tumorschmerz

\section{Zusammenfassung}

Ziel: Erfassung von Synchronisations- und Koordinationsreaktionen der Herzfrequenz (HF) auf einen musikalischen Rhythmus. Design: Randomisierte Pilotstudie. Probanden: $28 \mathrm{~Pa}-$ tienten mit chronischen Tumorschmerzen in einer stabilen Krankheitsphase. Intervention: 14tägiges Training einer für die Erleichterung des Einschlafens konzipierten Entspannungstherapie (ET) mit einer 30minütigen wiegenliedartigen, rhythmisch dominierten Musik allmählich abnehmenden Tempos. Kein Training in der Kontrollgruppe. Zielkriterien: Kontinuierliche Messung der Herzfrequenz und Vergleich mit der musikalischen Schlagfolge am Tag 1 und 15. Analyse des Synchronisationsgrades, d.h. der Koordination von Systole und musikalischem Schwerpunkt (1. Schlag im 6/8-Takt alla breve). Beobachtung des Einschlafverhaltens und Messung der subjektiven Bewertung der ET sowie der Schmerzintensität mittels "verbal rating scales». Dokumentation des Analgetikaverbrauchs. Ergebnisse: Unter der ET kam es bei Trainierten zur zunehmenden Synchronisation und Koordination der HF mit der musikalischen Schlagfolge. Bei einem musikalischen Tempo zwischen 48 und 42 Schlägen pro min bestand eine besonders stabile 2:3-Synchronisation. Patienten, die die ET subjektiv am angenehmsten empfanden und weniger Analgetika benötigten, zeigten tendenziell den höchsten Synchronisationsgrad. Die Einschlafhäufigkeit nahm unter ET zu, der Analgetikaverbrauch ab. Schlussfolgerungen: Musik mit wiegenliedartiger Struktur bewirkt innerhalb bestimmter Tempobereiche trainierbare Synchronisationen des Herzrhythmus, die mit der Entstehung und der Intensität einer Entspannungsreaktion funktionell verknüpft sind. Eine Verbesserung der Mess- und Dokumentationsmethoden ist erforderlich.

\section{Key Words}

Music therapy - Relaxation therapy · Rhythm interactions · Sleep disturbances $\cdot$ Cancer pain

\section{Summary}

Investigations into Synchronisation of Heart Rate and Musical Rhythm in a Relaxation Therapy in Patients with Cancer Pain

Objective: Registration of the influence of musical rhythm on synchronisation and coordination of heart rate. Design: Randomized pilot study. Probands: 28 patients with chronic cancer pain in a stable phase of the disease. Intervention: 14-day training of a relaxation therapy designed for improving the falling asleep, including a 30-minute lullaby-like, rhythmically dominated music with gradually decreasing tempi. No training in the control group. Outcome Measures: Continuous registration of heart rate and comparison with musical beat on day 1 and 15. Analysis of the degree of synchronisation, i.e. the coordination of systole and musical central time point (1st beat of the 6/8 time alla breve). Recording of the time of falling asleep and registration of the patient's subjective evaluation of the relaxation therapy and the pain intensity using verbal rating scales. Documentation of the use of analgetics. Results: Under the relaxation therapy trained patients showed an increasing synchronisation and coordination of heart rate and musical beat. At a musical tempo between 48 and 42 beats per min a very stable $2: 3$ synchronisation occurred. Trained patients who reported the best relaxing and analgetic effects showed the highest degree of synchronisation. Relaxation therapy led to an improvement of falling asleep and to a decrease in consumption of analgetics. Conclusions: Lullaby-like music within a special range of tempi can induce a trainable synchronisation of heart rate, functionally associated with the formation and intensity of a relaxation reaction. Further investigations are promising, however, substantial improvements in the measurement and documentation methods are needed.

$\begin{array}{ll}\text { KARGER } & \oplus 1999 \text { S. Karger GmbH, Freiburg } \\ \begin{array}{l}\text { Fax +497614520714 } \\ \text { E-mail kargergmbh@aol.com } \\ \text { www.karger.com }\end{array} & \begin{array}{l}\text { Accessible online at: } \\ \text { http://BioMedNet.com/karger }\end{array}\end{array}$

Priv.-Doz. Dr. med. Uwe Reinhardt 


\section{Einleitung}

Musik wird heute in verschiedenen Bereichen der Medizin eingesetzt. Dabei sind trotz zahlreicher Forschungsaktivitäten grundlegende Wirkungszusammenhänge weiterhin unklar. Neurophysiologische und psychologische Befunde zum Musikerleben deuten darauf hin, dass Musik zu einer Beeinflussung thalamisch-hypothalamischer und limbischer Strukturen führt, mit der Folge einer emotionalen Aktivierung und Veränderung biologischer Rhythmen [1].

Die therapeutische Musikanwendung erfolgt in der Regel als «Musiktherapie», wobei unter diesem Begriff allerdings sehr verschiedene Methoden und Verfahren subsummiert werden. Diese gehen von unterschiedlichsten konzeptionellen, theoretischen und weltanschaulichen Überlegungen aus, so dass innerhalb des Fachgebietes Definitions- und Abgrenzungsfragen intensiv diskutiert werden [2].

Bei sogenannten rezeptiven Musiktherapien werden musikalische Wirkungen ausschliesslich über das Anhören von Musik angestrebt. In diesem Rahmen erfolgt der Musikeinsatz überwiegend als «Musik in der Therapie» [3] und ist dabei Bestandteil integrativer therapeutischer Konzepte zur Unterstützung bzw. Erleichterung anderer Therapieformen, besonders der Psychotherapie, körperorientierter Verfahren oder bei pflegerischen Problemen z. B. in der Palliativmedizin [4].

Wie zahlreiche Beispiele aus der Anästhesie, der Intensivmedizin und der Psychiatrie belegen, kann insbesondere rhythmisch betonte «Musik» aber auch ohne den vermittelnden und aufarbeitenden Einfluss eines Therapeuten «als Therapie» wirken [5-7]. Es werden in diesem Zusammenhang weitgehend einheitliche Effekte beschrieben, die offenbar auch ohne stärkere Berücksichtigung der für Musikwirkungen sonst entscheidenden individuellen Faktoren, wie aktuelle Stimmungslage, Hörerfahrungen, Vorlieben usw. zu erzielen sind, so dass die Vermutung naheliegt, dass am Zustandekommen dieser Wirkungen Interaktionen des musikalischen Rhythmus mit körpereigenen Rhythmen einen wesentlichen Anteil haben.

In der vorliegenden Arbeit wurde im Rahmen eines Pilotprojektes untersucht, ob beim Anhören rhythmisch prägnanter Musik Veränderungen der Herzfrequenz auftreten, die als Wirkung des musikalischen Rhythmus zu deuten sind.

Dazu kam ein Entspannungsverfahren zum Einsatz, das zur Unterstützung des Einschlafvorganges entwickelt wurde und als wesentliches Element Musik mit einem dominanten, wiegenliedartigen, gleichmässigen Rhythmus enthält, dessen Tempo sich allmählich verlangsamt. Um zu prüfen, ob durch die Anwendung der Entspannungstherapie auch klinisch nutzbare Effekte zu erzielen sind, erfolgten die Untersuchungen an Patienten mit tumorbedingten Schmerzen.

Tumorschmerzen besitzen eine erhebliche psychosoziale Dimension: Die Schmerzintensität wird wesentlich durch Faktoren wie Isolation, Angst, Depressionen und Schlaflosigkeit beeinflusst, wobei sich diese Symptome im Sinne eines Circulus vitiosus gegenseitig verstärken (Abb. 7). Somit war zu vermuten, dass eine durch die Entspannungstherapie ausgelöste Verbesserung des Einschlafens auch zu einer Verbesserung der Schmerzsituation führen kann.

\section{Methoden}

\section{Design und Durchführung}

Die Untersuchung war als prospektive randomisierte Studie konzipiert. In die Studie wurden 28 Patienten (15 Männer und 13 Frauen) mit inkurablen, metastasierten Tumoren des Pankreas, der Prostata, der Mamma sowie des Magens im Alter von 36 bis 74 Jahren aufgenommen, bei denen chronische tumorbedingte Schmerzen bestanden. Voraussetzung für die Aufnahme in die Studie war eine stabile Krankheitssituation ohne rasche Krankheitsprogredienz und ohne belastende diagnostische oder therapeutische Eingriffe. Die Patienten waren unter einer (nach Einschätzung des Therapeuten) bestmöglichen analgetischen Basistherapie überwiegend schmerzarm und behandelten auftretende Schmerzspitzen mit Selbstmedikationen von unterschiedlicher Analgetika. Die Applikationshäufigkeit vor dem Versuchszeitraum sollte mindestens 2 bis 3 Selbstmedikationen pro 24 Stunden betragen und eine bestehende abendliche bzw. nächtliche Einstellung auf Sedativa oder Hypnotika sollte nicht verändert werden. Ausschlusskriterien waren Fieber, Anämie (Hb unter $12 \mathrm{~g} / \mathrm{dl}$ ) und kardiopulmonale Funktionsstörungen.

Die Patienten wurden per Losentscheid einer Trainingsgruppe (8 Frauen, 6 Männer im Alter von 44 bis 74 Jahren) und einer Kontrollgruppe (5 Frauen, 9 Männer im Alter von 36 bis 70 Jahren) zugeordnet.

Die Patienten der Therapiegruppe wurden aufgefordert, sich 13 Tage lang abends vor dem Einschlafen die Musikkassette mit dem aufgezeichneten Entspannungsverfahren anzuhören. Dazu wurde ihnen bei Bedarf ein transportables, selbstabschaltendes Wiedergabegerät ausgehändigt. Bei allen Patienten erfolgten Messungen am Tag 1 und 15 unter kontrollierten Bedingungen (s. u.). Die Kontrollgruppe trainierte das Entspannungsverfahren im dazwischenliegenden Zeitraum nicht.

Charakterisierung des Entspannungsverfahrens «Ein Weg in den Schlaf» (Edel Records 0001572CCC, Eterna 729195)

Zum Einsatz kam eine Entspannungstherapie (ET) von 30 min Länge, die für Kinder in Gemeinschaftseinrichtungen entwickelt wurde, um ihnen das Einschlafen während einer von der Hausordnung vorgesehenen Mittagsruhe zu erleichtern. Dabei handelt es sich um ein durchkomponiertes Stück für Kammerorchester mit Synthesizer, Mezzosopran und Sprecher, in das ein Wiegenlied, eine kleine bildhafte Erzählung, einige Ruhe- und Wärmesuggestionen sowie lyrische Szenen aus dem Phantasus von Arno Holtz eingeflochten sind. Der Charakter der musikalischen Substanz ist anfangs - die Tagesaktivität aufnehmend - lebhaft, dicht und vielschichtig und wird zunehmend in eine einfache, von einer Harfe vorgetragene, oft wiederkehrende musikalische Phrase aufgelöst. Prägend ist ein durchgehender, wiegender Rhythmus im 6/8-Takt alla breve mit einem Metrum von anfangs 5658 Schlägen pro min, gefolgt von einem allmählichen Ritardando, so dass nach 10 min ein Tempo von Metronomzahl 44 und nach 20 min von 36 erreicht ist.

Der Entspannungszustand wird am Ende des Stückes nicht (wie beispielsweise beim Autogenen Training) zurückgenommen, sondern Ziel ist das auch verbal suggerierte - Einschlafen.

\section{Beurteilung}

Die Messung der Herzfrequenz erfolgte mittels handelsüblicher Überwachungsgeräte der Firmen MLW und Siemens nach 10 min körperlicher Ruhe am liegenden Patienten kontinuierlich am Tag 1 und am Tag 15 des Versuchszeitraumes jeweils zwischen 18 und 21 Uhr. 
Die Probanden, die das Entspannungsverfahren durchführten, sollten protokollieren, ob sie am Vorabend unter dem Anhören eingeschlafen waren oder ob sie das Abschalten des Gerätes wahrgenommen hatten. Die Ermittlung des Einschlafzeitpunktes erfolgte durch Rekapitulation der letzten wahrgenommenen Textstelle.

Die Erfassung des subjektiven Schmerzempfindens erfolgte mittels einer 6stufigen «verbal rating scale» (VRS) nach Jensen [9]: keine Schmerzen (Grad 1), sehr leichte (Grad 2), leichte ( Grad 3), mässige (Grad 4), starke (Grad 5), sehr starke Schmerzen (Grad 6), wobei die Patienten jeweils am Morgen retrospektiv die Schmerzsituation des vergangenen Tages einzuschätzen hatten. Ausserdem wurden die Patienten aufgefordert, die Anzahl der zusätzlich erforderlichen Analgetikaapplikationen pro Tag zu protokollieren. An Hand einer weiteren VRS wurde die subjektive Bewertung des erreichten Entspannungszustandes erfragt.

\section{Statistik}

Die statistische Beurteilung erfolgte mit dem t-Test für abhängige und unabhängige Stichproben.

\section{Ergebnisse}

Alle Patienten beendeten die Studie protokollgemäss. Unerwünschte Begleitreaktionen der Entspannungstherapie wurden nicht beobachtet bzw. berichtet. Alle Patienten der Trainingsgruppe hatten die Entspannungstherapie während des Versuchszeitraumes mindestens $10 \mathrm{mal}$ angewendet.

\section{Verhalten der Herzfrequenz (HF)}

Unter der ET kam es zu einer Abnahme der HF von durchschnittlich 78 auf minimal 65 Schläge pro min (Abb. 1). Dabei unterschei- det sich der Kurvenverlauf der Patienten, die Entspannungstherapie trainierten («Trainierte» T) zwischen der 8. und 11. min der ET signifikant $(p=0,01)$ von demjenigen der Patienten, die das Verfahren nicht anwendeten («Untrainierte» $U$ ).

Dargestellt sind die Verhältnisse am Tag 15 des Versuchszeitraumes. Die Kurvenverläufe der Untrainierten von Tag 1 und Tag 15 boten keine signifikanten Unterschiede (nicht dargestellt).

Betrachtet man die Relation von HF und musikalischem Metrum (Abb. 2), so zeigt sich, dass dieser Quotient bei den Trainierten zwischen der 6. und 11. min der ET nahezu konstant bei 1,5 liegt. Dies entspricht einer Frequenzsynchronisation von $3: 2$, die hier also auch während der Verlangsamung des Tempos der Musik von 48 Schlägen pro min $(\mathrm{MM}=48)$ auf $\mathrm{MM}=40$ erhalten bleibt.

Ein zweites Plateau bei einem Quotienten von 2,0, entsprechend einer Synchronisation von 2:1, ist am Ende der ET zu beobachten, nachdem das musikalische Tempo auf 34 Schläge pro min abgefallen war.

Synchronisationsgrad von Herzfrequenz und musikalischem Rhythmus

Als optimale Synchronisation wurde das gleichzeitige Eintreffen von Systole und musikalischem Schwerpunkt, d. h. dem 1. Schlag des 6/8-Taktes, angesehen (Abb. 3); die prozentuale Häufigkeit dieses Ereignisses wurde während einer 30taktigen Periode bestimmt. Tabelle 1 dokumentiert die Ergebnisse der Messungen ab der 10. und 28. min der ET; hier lagen bei den Trainierten je-

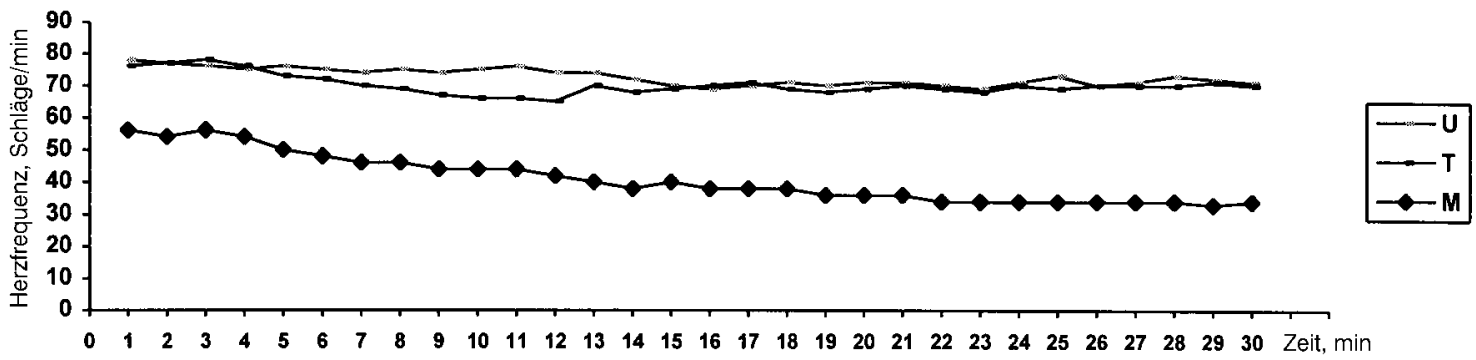

Abb. 1. Metrum der ET-Musik (M) und Verhalten der Herzfrequenz bei untrainierten (U) und trainierten Patienten (T) während der ET am Tag 15. Signifikant $(\mathrm{p}=0,01)$ niedrigere Herzfrequenz zwischen der 8. und 11. min durch Synchronisation mit dem musikalischen Rhythmus. Maximale Standardabweichung in diesem Zeitraum bei den Trainierten $\pm 1,8$, bei Untrainierten $\pm 3,0$ (minimale Standardabweichungen $\pm 1,4$ bzw. $\pm 1,8$ )

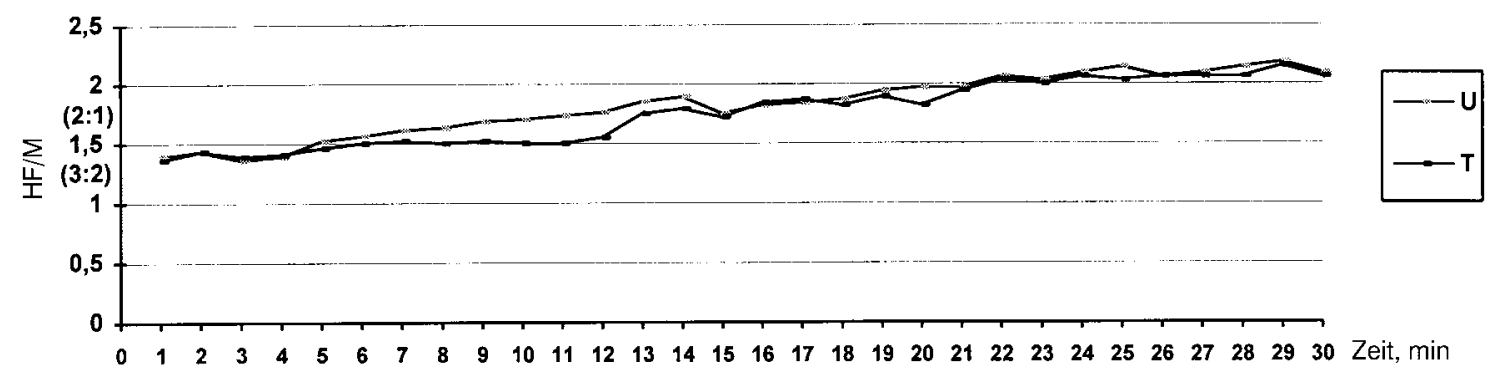

Abb. 2. Verhalten des Quotienten aus Herzfrequenz (HF) und musikalischem Metrum (M) unter ET bei untrainierten (U) und trainierten Patienten (T) am Tag 15. 


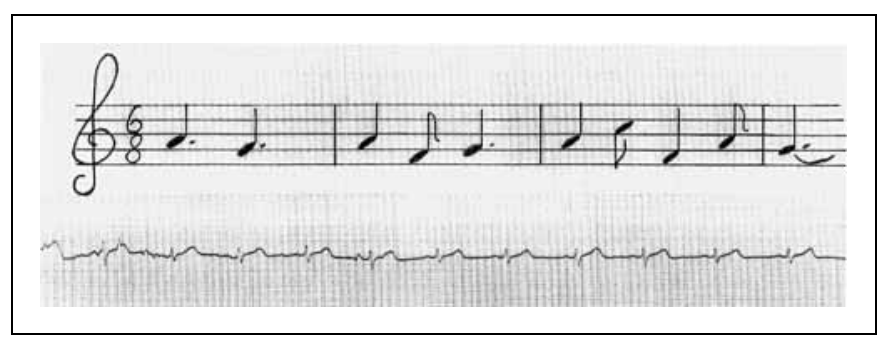

Abb. 3. 3:2-Synchronisation von Herzaktionen und musikalischem Schlag Optimaler Synchronisationsgrad von musikalischem Schwerpunkt (1. Schlag im $6 / 8$-Takt alla breve) und R-Zacke (Systole).

Tab. 1. Häufigkeit optimaler Synchronisationen von HF und musikalischem Rhythmus nach 10 und 28 min der ET bei untrainierten (U), allen Trainierten $(\mathrm{T})$ sowie bei den (trainierten) Patienten mit der deutlichsten Schmerzreduktion $(\mathrm{A})$ und der positivsten Bewertung der ET (E)

\begin{tabular}{lcclll}
\hline Patientengruppe & \multicolumn{2}{l}{ Synchronisationsgrad } \\
\cline { 2 - 3 } & \multicolumn{2}{l}{ Tag 1} & & Tag 15 & \\
\cline { 2 - 3 } \cline { 5 - 6 } & $10^{\prime} \mathrm{ET}$ & $28^{\prime} \mathrm{ET}$ & & $10^{\prime} \mathrm{ET}$ & $28^{\prime} \mathrm{ET}$ \\
\hline $\mathrm{U}$ & 8 & 10 & 14 & 10 \\
$\mathrm{~T}$ & 9 & 8 & 28 & 20 \\
$\mathrm{~A}$ & 12 & 10 & 32 & 18 \\
$\mathrm{E}$ & 11 & 11 & 36 & 24 \\
\hline
\end{tabular}

weils relativ stabile $3: 2$ - bzw. 2:1-Synchronisationen vor. Zusätzlich zu den Ergebnissen der Gesamtgruppe der Trainierten und Untrainierten wurden als Subgruppenanalyse die Verhältnisse bei den Trainierten dargestellt, die die ET subjektiv als «sehr entspannend» beschrieben $(\mathrm{E}, \mathrm{n}=9)$ und bei den Patienten, die die Analgetikaeinnahmen am meisten reduzierten (A, $\mathrm{n}=7)$.

Die Daten zeigen, dass die Qualität der Synchronisation durch regelmässige Anwendung der ET zunahm und dass sowohl ein ausgeprägtes subjektives Entspannungsgefühl als auch ein guter analgetischer Effekt der ET mit der Fähigkeit zur Ausbildung eines hohen Synchronisationsgrades verknüpft waren.

\section{Einschlafverhalten und subjektive Einschätzung der Entspannungs- therapie}

Abbildung 4 zeigt, dass bei der ersten Sitzung 11\% der Patienten unter der ET einschliefen. Die Einschlafrate steigerte sich unter dem Training der ET und erreichte einen Maximalwert von 43\%. Der Einschlafzeitpunkt lag bei den von den Patienten selbst dokumentierten Sitzungen zwischen der 5. und 18. min, bei den vom Untersucher beobachteten Fällen erfolgte das Einschlafen zwischen der 4. und 13. min der ET.

Mittels der 6stufigen VRS schätzten nach der 1. Sitzung (Tag 1) $43 \%$ der 28 Patienten die ET als «sehr entspannend»(Grad 1$)$, $36 \%$ als «entspannend» $(\operatorname{Grad} 2)$ und $21 \%$ als «etwas entspannend» (Grad 3) ein. Am Tag 15 kam es zu folgenden Beurteilun- gen bei den Untrainierten: «sehr entspannend» 57\%, «entspannend» $43 \%$. Bei den Trainierten fiel auf, dass 3 Patienten $(21 \%)$ die ET nach Abschluss der Therapie als «eher nicht entspannend» (Grad 4) empfanden, von den verbleibenden 11 Patienten beurteilten $9(64 \%)$ die ET als «sehr entspannend», zwei Patienten als «entspannend».

\section{Schmerzverhalten}

Die regelmässige Anwendung der ET führte zu einer signifikanten Abnahme der zusätzlich benötigten Analgetikamedikation von anfangs durchschnittlich 2,9 Einnahmen auf 2,0 Selbstmedikationen pro Tag $(p=0,05)$. Hingegen zeigte der Analgetikaverbrauch in der Kontrollgruppe eher eine leicht steigende Tendenz (Abb. 5).

Auf die subjektive Beurteilung der Schmerzsituation mittels der VRS wirkte sich das Training der ET nicht aus (Abb. 6).

\section{Diskussion}

Dass Musik die Herzfrequenz, den Blutdruck, die Atmung, die Hirndurchblutung usw. beeinflusst, bedarf keiner besonderen Betonung für den, der in jedem Affekt eine koordinierte animalischvegetative Gesamtreaktion erblickt, deren motorische Äusserung lediglich unterdrückt ist durch anerzogene Selbstbeherrschung [10]. Wirkt Musik ausschliesslich über die Affektivität, dann ist eine unmittelbare, sozusagen gesetzmässige Verknüpfung bestimmter Musikformen mit bestimmten vegetativen Folgen nicht zu erwarten, da die emotionale Ausgangslage über die Reaktion auf ein Musikstück entscheidet. Für den Fall, dass weitere Wirkungswege existieren, würden bestimmte Musikwirkungen eher vorhersagbar und auch erklärbar, denkt man z.B. an die mitreissenden Effekte des Trommelns und mancher Marsch- und Tanzmusik, denen man sich trotz einer innerlich ablehnenden Haltung nicht entziehen kann.

Schon seit langem ist bekannt, dass attraktive Rhythmen als externe Zeitgeber körpereigene biologische Rhythmen beeinflussen können. Musik kann als solch rhythmischer Impulsgeber selbsterregte Schwingungen wie den Herzrhythmus «einfangen» und «mitnehmen», falls die beiden Frequenzen initial nicht zu sehr abweichen und bestimmte Grenzen nicht überschritten werden [11].

Biedermann [12] wies nach, das solche Konditionierungen der Herzfrequenz auch unabhängig von der subjektiven Bewertung der entsprechend rhythmischen Musik erreichbar sind. Diese Beobachtung blieb nicht unwidersprochen, da andere Arbeitsgruppen zeigten, dass die Wirkungen des musikalischen Rhythmus im Rahmen des komplexen Musikgeschehens auch von der emotionalen Ausgangslage bzw. von Vorlieben und Vorerfahrungen der Versuchspersonen abhängen $[13,14]$. Ursache für diese diskrepanten Befunde könnte die Stärke bzw. Intensität sein, mit der der Rhythmus musikalisch vermittelt wird. 


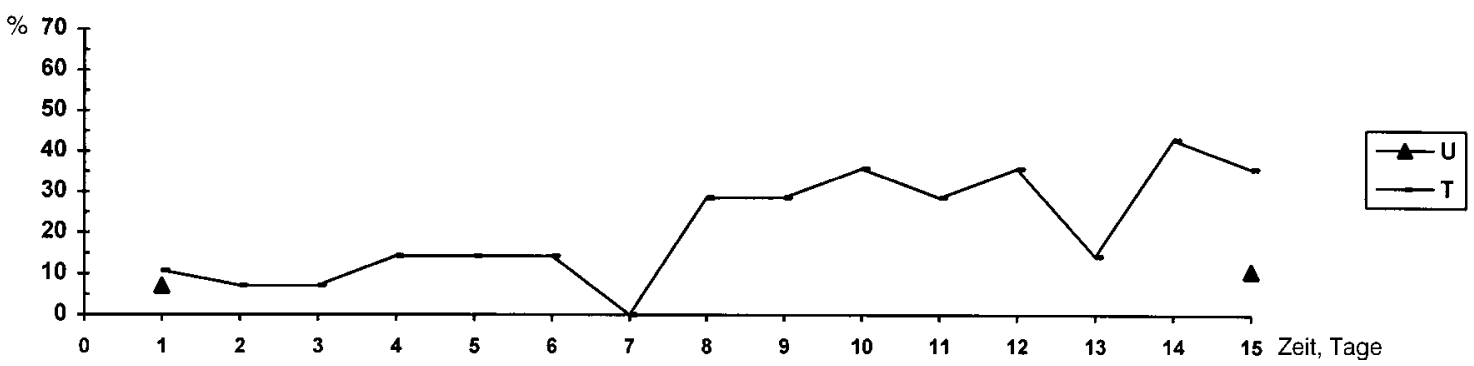

Abb. 4. Prozentualer Anteil der während des Anhörens der ET eingeschlafenen Patienten: Trainierte (T), Untrainierte (U).

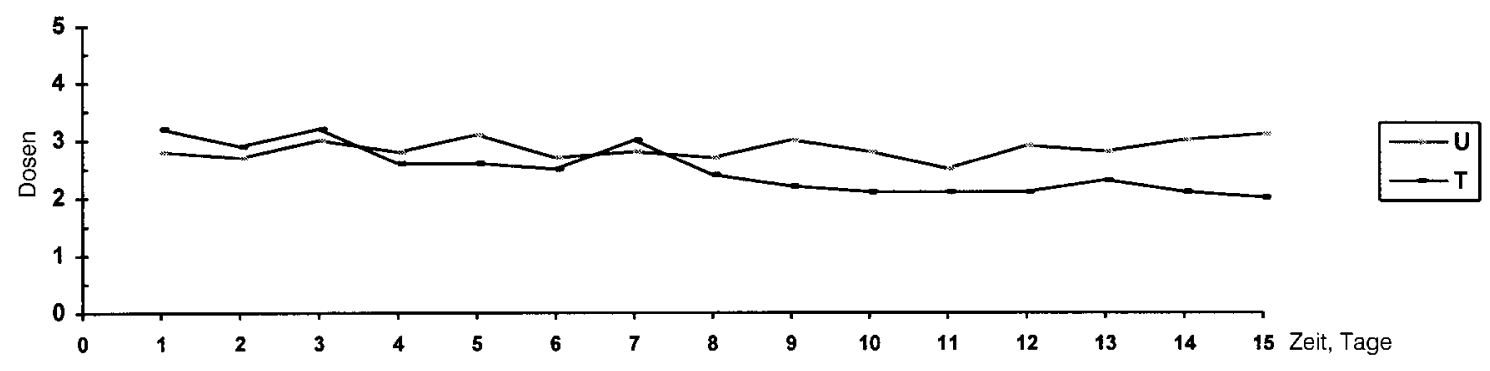

Abb. 5. Durchschnittlicher Analgetikaverbrauch (Anzahl zusätzlich zur Basismedikation eingenommener Dosen) bei Tumorpatienten mit (T) und ohne (U) tägliches Training der Entspannungstherapie. Signifikanter $(\mathrm{p}=0,05)$ Unterschied ab Tag 9 . Standardabweichungen zwischen $\pm 0,4$ und $\pm 0,9$.

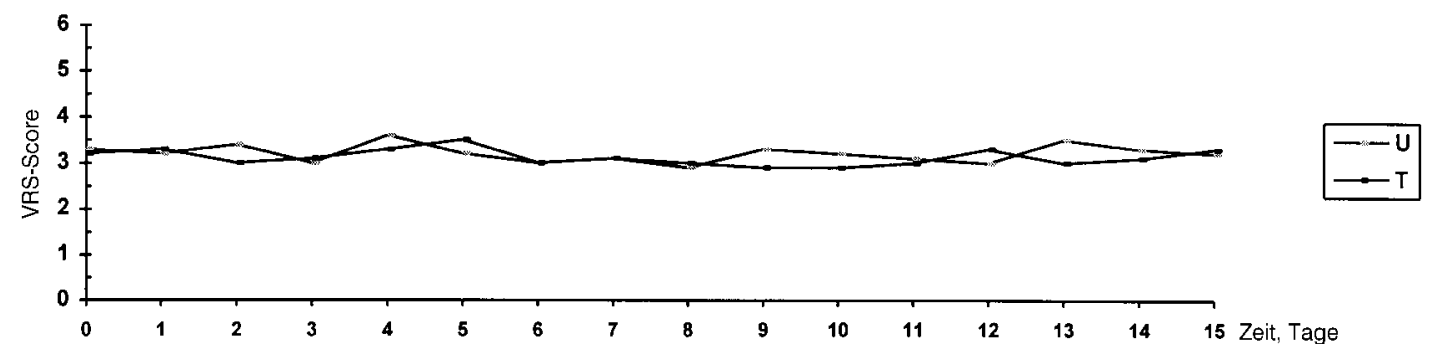

Abb. 6. Selbsteinschätzung der Schmerzsituation mittels einer VRS bei Tumorpatienten mit (T) und ohne (U) tägliche ET.

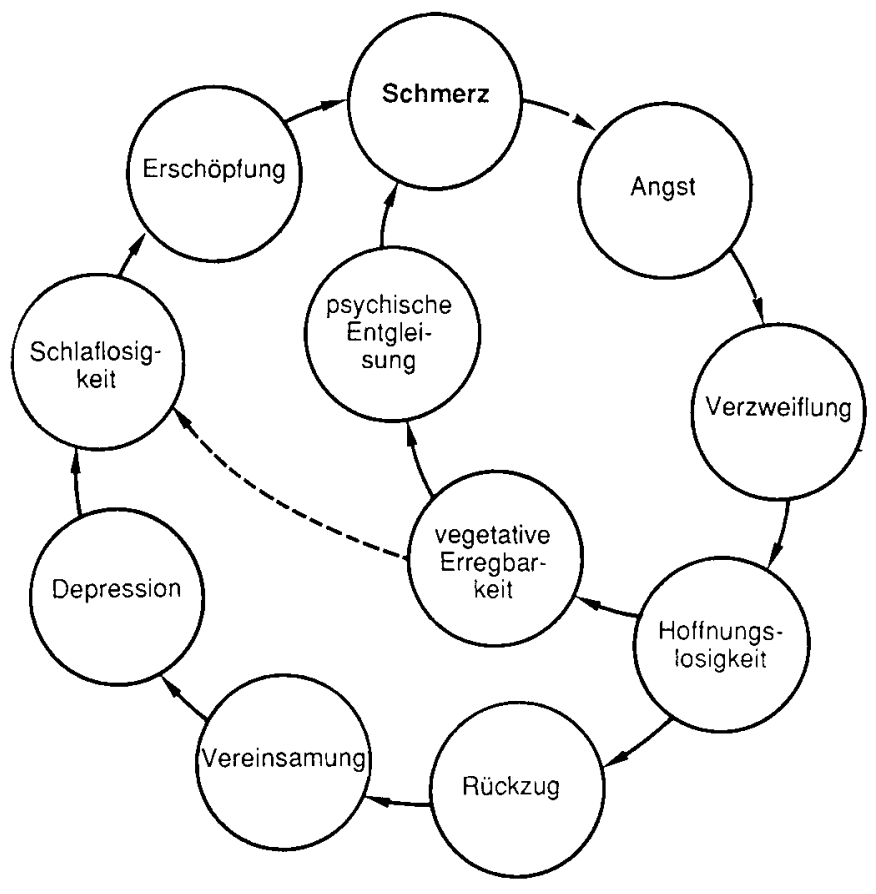

Abb. 7. «Schmerzspirale» tumorbedingter Schmerzen [8].
Synchronisationsvorgänge von Herzfrequenz und externem Zeitgeber wirken sich auch auf die Koordination von Herzfrequenz und Atemfrequenz aus [15]. Offenbar lässt sich eine Frequenzsteigerung im Sinne eines «Puls- und Atemdriving» [16] dabei musikalisch leichter erreichen als eine Frequenzsenkung. Diese gelang in Untersuchungen von Saperston [17] mit «physiologisch interaktiver Musik» nur mit Musikstücken, deren Tempo der Herzfrequenz der Versuchspersonen angepasst war.

In der eigenen Untersuchung lag die musikalische Schlagfolge von Anfang an deutlich unter der Pulsfrequenz der Patienten. Trotzdem kam es relativ rasch und einheitlich zu einer Herzfrequenzabnahme, was schon allein damit erklärt werden könnte, dass die Untersuchungen im Rahmen einer Entspannungstherapie erfolgten, die per se eine Abnahme der Herzschlagfolge anstrebt.

Dafür, dass zusätzlich eine direkte Rhythmusbeeinflussung vorliegt, spricht, dass sich nach wiederholtem Anhören der Musik bei einem Grossteil der Patienten an bestimmten Abschnitten des Untersuchungsablaufes stabile Synchronisationen zwischen dem musikalischen Rhythmus und der Herzfrequenz einstellten, die auch bei weiterer Senkung des musikalischen Tempos über einen längeren Bereich erhalten bleiben. 
Am ausgeprägtesten ist dies zwischen der 6. und 12. min, wo bei einem musikalischen Tempo zwischen 48 und 42 Schlägen pro min eine eindeutige Tendenz zur 3:2-Synchronisation besteht (Abb. 2, 3). Danach führt die weitere Verlangsamung des musikalischen Tempos schliesslich auch bei den trainierten Patienten nicht mehr zu einer konsekutiven Herzfrequenzverlangsamung, sondern die Synchronisation geht wieder verloren. Erst bei einem Tempo von 34 Schlägen pro min lässt sich erneut eine Tendenz zur Synchronisation, jetzt im Verhältnis 2:1, nachweisen. Diese Beobachtung bestätigt die Ergebnisse anderer Arbeitsgruppen [18, 19], dass sich Synchronisationen nur bei bestimmten Frequenzen beobachten lassen, und zeigt, dass die Rhythmusinteraktionen nicht nur im Verhältnis $1: 1$, sondern auch in verschiedenen anderen Verhältnissen möglich sind.

Das stärkste Indiz dafür, dass es sich bei den Veränderungen der Herzfrequenz nicht nur um normale Veränderungen im Rahmen emotionaler Entspannung, sondern um Reaktionen auf einen attraktiven Rhythmus handelt, ist die bei einem grossen Teil der Patienten zu beobachtende Tendenz, dass die Systole zunehmend zeitgleich auf den musikalischen Schwerpunkt, d.h. mit dem 1. Schlag im 6/8-Takt, synchronisiert wird.

Auch wenn die kleinen Patientenzahlen, insbesondere bei der Betrachtung der Subgruppen, zuverlässige Aussagen nicht zulassen, kann doch vermutet werden, dass ein Zusammenhang zwischen der Synchronisationsbereitschaft des Herzschlages und der Fähigkeit zur vegetativen und psychischen Entspannung besteht und dass sich diese Fähigkeit trainieren lässt, falls nicht organische oder psychische Erregungen so dominant sind, dass sie eine Relaxation verhindern.

Die in der vorliegenden Arbeit eingesetzte Musik benutzt typische Charakteristika von Wiegenliedern.

Aus Untersuchungen von Last und Kneutgen [20] ist bekannt, dass sich die Wiegenlieder verschiedener Kulturkreise der Menschheit rhythmisch, melodisch und metrisch weitgehend ähneln und dass sie auch beim Erwachsenen noch schlafanstossend wirken können.

Ein wiegender 6/8-Takt alla breve im Tempo von 42 bis 44 Schlägen pro min, wie er in der verwendeten Entspannungstherapie nach 10 min erreicht ist, entspricht gefühlsmässig recht genau unserer Wiegenliedvorstellung.

Bei den trainierten Patienten lag zu diesem Zeitpunkt nicht nur eine stabile Synchronisation vor, sondern es fand sich auch der weitaus höchste Koordinationsgrad zwischen der Systole und dem musikalisch-rhythmischen Geschehen (Tab.1). Möglicherweise liegt hier ein «archetypischer» Rhythmus- und Tempobereich vor, in dem die Ankopplungsbereitschaft der Herzfrequenz besonders gross ist.

Um diesen Zeitpunkt wurde auch von den meisten Patienten, die unter der Entspannungstherapie einschliefen, dieses («dramaturgisch» eigentlich erst nach 25 min vorgesehene) Ziel erreicht. Interessant wäre es deshalb zu prüfen, ob die ET ihr «Einschlafziel» nicht noch besser erreichte, wenn dieses musikalische Tempo beibehalten würde.

Im Vergleich $\mathrm{zu}$ «richtigen» Wiegenliedern ist ein Tempo von 44 Schlägen pro min allerdings zu langsam. Dies dürfte am ehesten mit der höheren Ruhefrequenz von Neugeborenen und Kleinkindern erklärbar sein, die für eine optimale Synchronisation bzw. Koordination einen schnelleren externen Zeitgeber benötigen.

Möglicherweise besitzt jede Altersstufe ihren Rhythmus- und Tempobereich, in dem die Ankopplungsbereitschaft der Herzfrequenz hoch und die Tendenz zur Ruhe und Geborgenheit vermittelnden harmonischen Koordination verschiedener Rhythmen besonders hoch ist. In der musiktherapeutischen Praxis wird diese Anpassung der rhythmischen Struktur an die Puls- und Atemfrequenz als wichtige Voraussetzung für eine optimale Wirksamkeit seit langem berücksichtigt [18].

Die allgemeine Erfahrung, dass ein erleichtertes Einschlafen über eine Verbesserung der psychischen Gesamtsituation auch das Schmerzempfinden beeinflussen kann, liess sich in dem Pilotprojekt an Hand der Verringerung zusätzlich benötigter Schmerzmedikationen bei den Patienten, die die Entspannungstherapie trainierten, bestätigen.

Die unter der Entspannungstherapie ausbleibenden Veränderungen in der subjektiven Schmerzbeurteilung mittels einer VRS deuten darauf hin, dass die potentiellen angstlösenden Wirkungen eines Entspannungsverfahrens angesichts der permanenten Bedrohung durch die Tumorerkrankung in den Hintergrund treten. Demgegenüber wurde die Verbesserung des Einschlafens als ausgesprochen wohltuend empfunden und trug zu einer signifikanten Einsparung an Analgetika bei. Günstig für die Compliance wirkte sich dabei aus, dass durch die Anwendung der Entspannungstherapie unmittelbar vor dem Einschlafen kein zusätzlicher Zeitbedarf entstand.

Weitere Untersuchungen zu dieser Thematik sind nicht nur an grösseren Patientenkollektiven erforderlich, sondern müssen unbedingt auch eine Verbesserung der Messmethodik einschliessen. Wichtig erscheint vor allem, Rhythmusanalysen prospektiv durchzuführen, so dass bereits das zu untersuchende musikalische Material digital und computergestützt produziert und aufgezeichnet wird, damit z.B. auch eine Veränderung der attraktiven Rhythmen, die parallele Dokumentation von musikalischen und biorhythmischen Vorgängen und die Betrachtung einzelner Phasen der Herz- und Atemaktionen problemloser möglich werden. Aus chronobiologischen Untersuchungen von Herzfrequenz und Atmung ist bekannt, dass erst die genauere Analyse der Phasenkoordination als Mass für die klinische Effizienz herangezogen werden kann [21].

Im Zusammenhang mit der klinischen Erfahrung, dass sich die Erlernbarkeit und die Wirksamkeit verschiedener Entspannungsverfahren durch die Einbeziehung von Musik verbessert [22], lassen die gewonnenen Ergebnisse vermuten, dass die beobachteten Vorgänge der Synchronisation und Koordination der Herzfrequenz nicht nur eine Bestätigung für die Anpassung eines biologischen Rhythmus an den musikalischen Rhythmus als externen Zeitgeber sind, sondern dass sie auch die Entspannungsreaktion unmittelbar beeinflussen. Für den praktischen therapeutischen Einsatz eröffnete sich damit die Möglichkeit, durch eine gezieltere Musikauswahl die Geschwindigkeit und die Effizienz von Entspannungsvorgängen zu erhöhen. 


\section{Literatur}

1 Müller-Busch HC: Schmerz und Musik. Jena, Fischer, 1997.

2 Bunt L: Musiktherapie. Weinheim, Beltz, 1998.

3 Bruscia KE: Defining Music Therapy. Spring City, Spring House Books, 1989.

4 Petzold H: Integrative Musiktherapie - eine Ausbildung mit klinischer, ästhetischer und psychotherapeutischer Schwerpunktbildung; in Müller L, Petzold H (Hrsg): Musiktherapie in der klinischen Arbeit. Jena, Fischer, 1997, pp 278-295.

5 Spintge R, Droh R: Musik-Medizin. Stuttgart, Fischer, 1992.

6 Möckel M, Störk T, Vollert J, Röcker L, Danne O, Hochrein H, Eichstädt H, Frei U: Stressreduktion durch Musikhören. Dtsch Med Wochenschr 1995;120:745-752.

7 Reinhardt U, Lange E: Musikwirkungen bei Depressiven. Psychiatr Neurol Med Psychol 1982;34:414-421.

8 Aulbert E, Hankemeier U: Systemische medikamentöse Schmerzbehandlung bei Krebskranken; in Aulbert E, Niederle N (Hrsg): Die Lebensqualität des chronisch Krebskranken. Stuttgart, Thieme, 1990, pp 131-146.

9 Jensen MP, Karoly P, Braver S: The measurement of clinical pain intensity: A comparison of six methods. Pain 1986;27:117-126.

10 Klumbies G: Psychotherapie in der Inneren und Allgemeinmedizin. Leipzig, Hirzel, 1974.

11 Bason BT, Celler BG: Control of the heart rate by external stimuli. Nature 1972;4:279-280.

12 Biedermann BR: Synchronizing music to heart-rate; in Bejjani FJ (ed): Current Research in Arts Medicine. Chicago, Chicago Review Press, 1993, pp 449-452.

13 Dainow E: Physical effects and motor responses to music. J Acoust Soc Am 1977;39:414-416.

14 Landreth JE, Landreth HF: Effects of music on physiological response. J Res Music Educ 1974;22:4-12.

15 Frank C: Musikrhythmen als möglicher Synchronisator für biologische Rhythmen? in Harrer G (Hrsg): Grundlagen der Musiktherapie und Musikpsychologie. Stuttgart, Fischer, 1982, pp 105-110.

16 Harrer G: Das «Musikerlebnis» im Griff des naturwissenschaftlichen Experiments; in Harrer G (Hrsg): Grundlagen der Musiktherapie und Musikpsychologie. Stuttgart, Fischer 1982, pp 3-54.

17 Saperston BM: Music based models for altering physiological responses; in Bejjani FJ (ed): Current Research in Arts Medicine. Chicago, Chiccago Review Press, 1993, pp 379-378.

18 Schwartz FJ: Music and medicine; in Bejjani FJ (ed): Current Research in Arts Medicine. Chicago, Chicago Review Press, 1993, pp 375-382.

19 Gardner WJ, Licklider JCR, Weisz AJ: Suppression of pain by sound. Science 1960;131:1583-1588.

20 Last G, Kneutgen J: Schlafmusik. Münch Med Wochenschr 1970;44:20112016.

21 Hildebrandt G: Reactive modifications of the autonomous time structure in the human organism. J Physiol Pharmacol 1991;42:5-27.

22 Schwabe C: Musiktherapie bei Neurosen und funktionellen Störungen. Jena, Fischer, 1974. 